\title{
Baryogenesis from neutron-dark matter oscillations
}

\author{
Torsten Bringmann* \\ Department of Physics, University of Oslo, Box 1048, NO-0371 Oslo, Norway \\ James M. Cline ${ }^{\dagger}$ \\ McGill University, Department of Physics, 3600 University Street, Montréal, Québec H3A2T8, Canada \\ Jonathan M. Cornell \\ McGill University, Department of Physics, 3600 University Street, Montréal, Québec H3A2T8, Canada, \\ and Department of Physics, University of Cincinnati, Cincinnati, Ohio 45221, USA
}

(Received 13 November 2018; published 19 February 2019)

\begin{abstract}
It was recently suggested that dark matter consists of $\sim \mathrm{GeV}$ particles that carry baryon number and mix with the neutron. We demonstrate that this could allow for resonant dark matter-neutron oscillations in the early Universe, at finite temperature, leading to low-scale baryogenesis starting from a primordial dark matter asymmetry. In this scenario, the asymmetry transfer happens around $30 \mathrm{MeV}$, just before big bang nucleosynthesis. We illustrate the idea using a model with a dark $U(1)^{\prime}$ gauge interaction, which has recently been suggested as a way of addressing the neutron lifetime anomaly. The asymmetric dark matter component of this model is both strongly self-interacting and leads to a suppression of matter density perturbations at small scales, allowing it to mitigate the small-scale problems of cold dark matter cosmology. Future cosmic microwave background experiments will be able to consistently probe, or firmly exclude, this scenario.
\end{abstract}

DOI: 10.1103/PhysRevD.99.035024

\section{INTRODUCTION}

One of the curious coincidences of the $\Lambda \mathrm{CDM}$ cosmological model is the rough similarity of the contributions from baryons and dark matter (DM) to the energy density, $\Omega_{b} \simeq 0.049$ versus $\Omega_{\mathrm{CDM}} \simeq 0.26$ [1], given that in typical scenarios of the early Universe they have completely different origins. An appealing feature of many models of asymmetric DM is that this coincidence could be due to DM having a mass at the GeV scale, like baryons, and a shared mechanism for the generation of the two asymmetries. This could be achieved through either a common "cogenesis" event that creates the required asymmetries in the dark and visible sector, or through an efficient sharing of asymmetries created in independent ways, though (dark) sphalerons, new renomalizable interactions, or higher-dimensional effective operators (for a review, see Ref. [2]). In particular, the baryon asymmetry of the Universe (BAU) could be fully explained by the

\footnotetext{
*torsten.bringmann@fys.uio.no

jcline@physics.mcgill.ca

;onathan.cornell@uc.edu
}

Published by the American Physical Society under the terms of the Creative Commons Attribution 4.0 International license. Further distribution of this work must maintain attribution to the author(s) and the published article's title, journal citation, and DOI. Funded by SCOAP. "darkogenesis" of an asymmetry in the dark sector that is subsequently transferred to the standard model (SM) sector. Reference [3] analyzes this scenario in terms of the lowest-dimension, gauge-invariant effective operators that would allow such a redistribution of a primordial asymmetry starting in the dark sector. For example, the operator $\chi u d d / \Lambda^{2}$ would induce roughly equal asymmetries between DM particles $\chi$ and quarks if it were in equilibrium at high temperatures. ${ }^{1}$

In this work, we consider a novel situation where the $\chi u d d / \Lambda^{2}$ coupling only arises below the QCD phase transition. Then it is replaced by the unique relevant operator that could connect Dirac DM to the SM, namely mass-mixing with the neutron,

$$
\mathcal{L}_{\text {mix }}=-\delta m \bar{n} \chi+\text { H.c. }
$$

Our purpose is to show that it can produce the baryon asymmetry at low temperatures $\sim 30 \mathrm{MeV}$, starting from a $\chi$ asymmetry (whose darkogenesis origin we do not try to specify here). ${ }^{2}$ This comes about by oscillations between $\chi$ and $n$, in analogy to neutrino oscillations, that are

\footnotetext{
${ }^{1}$ This example requires $m_{\chi}<m_{n}$, since it would otherwise allow the DM to decay into quarks.

${ }^{2}$ We note that the residual symmetric baryon component is negligible by this time; see, e.g., Fig. 5 of Ref. [4].
} 
resonantly enhanced by finite-temperature effects. Oscillations of neutrons to a mirror-sector partner have been studied extensively in Refs. [5-8]; however, to our knowledge these oscillations have never been considered as a means for baryogenesis. This adds a new option to the two broad classes of asymmetry transfer mechanisms discussed so far-namely, (dark) sphalerons (e.g., Refs. [3,9-16]) and renormalizable interactions or higher-dimensional operators (e.g., Refs. [3,13,17-23]). Similar ideas for producing the baryon asymmetry at low temperatures via oscillations of SM baryons have been recently suggested in Refs. [4,24].

Recently, motivation for the operator in Eq. (1) has come from a quite different direction. It was suggested [25] that mixing between the neutron and DM could resolve a longstanding discrepancy between determinations of the neutron lifetime from decay-in-flight ( $p$ appearance) versus bottle ( $n$ disappearance) measurements, by having a small dark decay channel [26]. The proposed decays $n \rightarrow \chi \gamma$ and $n \rightarrow \chi e^{+} e^{-}$were quickly ruled out by experimental searches [27,28], suggesting a completely hidden channel, like $n \rightarrow \chi \gamma^{\prime}$, where $\gamma^{\prime}$ is a dark photon. Even this model is ruled out by neutron star properties [29-32], unless repulsive $\chi$ self-interactions are strong enough, requiring

$$
\frac{m_{\gamma^{\prime}}}{g^{\prime}} \lesssim(45-60) \mathrm{MeV}
$$

to be satisfied [33]. (The uncertainty in the bound is due to the unknown nuclear equation of state.) This scenario is highly constrained by a number of observables sensitive to $\gamma^{\prime}$, including big bang nucleosynthesis (BBN), the cosmic microwave background (CMB), DM direct detection, supernovae, and structure formation effects from DM self-interactions.

In the present work, we will show that it is possible to explain both the baryon asymmetry and the neutron decay anomaly within the same model, while at the same time evading all cosmological bounds, if $m_{\gamma^{\prime}} \sim 60 \mathrm{keV}$ and an additional species of dark radiation $\nu^{\prime}$ is introduced to enable the decay $\gamma^{\prime} \rightarrow \nu^{\prime} \bar{\nu}^{\prime}$. This entails moderate tension with $\mathrm{BBN}$ and $\mathrm{CMB}$ limits on extra radiation species, implying that it may be testable already in the near future. Alternatively, larger $m_{\gamma^{\prime}}$ consistent with $\gamma^{\prime} \rightarrow e^{+} e^{-}$, and hence without the need of introducing the additional degrees of freedom contributed by $\nu^{\prime}$, can be accommodated if we disregard the neutron lifetime anomaly.

\section{THEORETICAL FRAMEWORK}

We consider the same model as in Ref. [33], with an elementary Dirac DM particle $\chi$ that carries baryon number

\footnotetext{
${ }^{3}$ It has also been proposed that neutron-mirror neutron oscillations could explain this puzzle [8].
}

and mixes with the neutron through the mass term [Eq. (1)], and a dark photon coupling to $\chi$ with strength $g^{\prime}$. In order to kinematically allow for the decay $n \rightarrow \chi \gamma^{\prime}$, the $n-\chi$ mass splitting must satisfy

$$
\Delta m \equiv m_{n}-m_{\chi}>m_{\gamma^{\prime}}
$$

However, there is also a subdominant decay channel $n \rightarrow$ $\chi \gamma$ in this case, suppressed by the dipole moment $\mu_{n}$ of the neutron. This imposes

$$
\Delta m<1.665 \mathrm{MeV}
$$

for the stability of ${ }^{9} \mathrm{Be}$, which is the most constraining nuclear decay [25]. We note that ${ }^{11} \mathrm{Be}$ has an even smaller neutron binding energy, $0.504 \mathrm{MeV}$ rather than $1.665 \mathrm{MeV}$, which would further strengthen this limit [34].

Another relevant parameter is the ratio of the temperatures in the two sectors,

$$
\xi=\frac{T_{\gamma^{\prime}}}{T}
$$

(or $T_{\nu^{\prime}} / T$ at later times where $\gamma^{\prime} \rightarrow \nu^{\prime} \bar{\nu}^{\prime}$ has already occurred). We will show how $\xi<1$ is determined by the decoupling temperature between the two sectors, which turns out to be independent of the details of the UV physics. The baryon asymmetry depends mainly on $\Delta m$ and the mixing mass $\delta m$, with weak dependence on the combination $g^{\prime} \xi$.

The dark photon mass must satisfy Eq. (3) to explain the neutron lifetime anomaly. We will further show that $\Delta m \lesssim$ $0.2 \mathrm{MeV}$ is required to simultaneously respect the limit from $n \rightarrow \chi \gamma$ and to obtain the observed baryon asymmetry. This bound kinematically forbids $\gamma^{\prime} \rightarrow e^{+} e^{-}$decays, which in turn makes the dark photon long lived, such that it overcloses the Universe [33,35]. To avoid this, we supplement the model with an inert light particle, dark radiation, taken to be a massless Dirac neutrino $\nu^{\prime}$ that couples to $\gamma^{\prime}$ with strength $Q_{\nu^{\prime}}^{\prime} g^{\prime}$ and charge $Q_{\nu^{\prime}}^{\prime} \neq 1$. This is anomaly free and allows for $\gamma^{\prime} \rightarrow \nu^{\prime} \bar{\nu}^{\prime}$, while the charge difference between $\nu^{\prime}$ and $\chi$ forbids mass mixing between the states, which would lead to proton decay.

A further requirement is that the symmetric component of the DM must be sufficiently diluted by annihilations. The channels $\bar{\chi} \chi \rightarrow \gamma^{\prime} \gamma^{\prime}, \nu^{\prime} \bar{\nu}^{\prime}$ turn out to be too inefficient, since we need a small gauge coupling $g^{\prime} \sim 10^{-3}$ to satisfy previous requirements. This can be overcome by introducing a heavy dark fermion $\psi$ with coupling $\bar{\chi} \phi \psi$ to the DM and the dark Higgs $\phi$, allowing for $\chi \bar{\chi} \rightarrow \phi \phi^{*}$ through the exchange of $\psi$.

Finally, one should avoid leakage of the primordial $\chi$ asymmetry into the SM sector at high temperatures, since this would generally produce too large a baryon asymmetry relative to the DM abundance. We point out a simple 
mechanism for enforcing this requirement in the present model, by adding a very weakly coupled interaction $\bar{\chi} \phi \psi^{\prime}$ involving a heavy Majorana dark sector fermion $\psi^{\prime}$.

\section{III. $\chi$ - $n$ OSCILLATIONS}

The oscillations of $\chi$ into neutrons are determined by a $2 \times 2$ Hamiltonian that includes the mass terms and thermal self-energy corrections from the forward scattering of the fermions on particles in the plasma. The most important such interactions are the elastic scattering of neutrons on pions and DM on dark photons.

The Hamiltonian is given by

$$
\mathcal{H}=\left(\begin{array}{cc}
\Delta E_{n}+m_{n} & \delta m \\
\delta m & \Delta E_{\chi}+m_{\chi}
\end{array}\right),
$$

where $\delta m$ is the mass mixing introduced in Eq. (1). The thermal energy correction $\Delta E_{n}$ to the neutron has been calculated in Refs. [36-40] using various techniques. Some of these results are plotted in Fig. 1 (left panel). The most reliable ones use dispersion relations, supplemented by experimentally measured scattering cross sections [36,37]. We have digitized the results of Ref. [37] as our estimate of $\Delta E_{n}$. Using thermal field theory techniques similar to Ref. [41] (see Appendix A for details), we find for the DM energy shift

$$
\Delta E_{\chi}=g^{\prime 2} \frac{T_{\gamma^{\prime}}^{2}}{8 m_{\chi}} .
$$

This result is also plotted, for $g^{\prime} \xi=1$, in Fig. 1 (right panel).

The combination $\Delta E_{n}-\Delta E_{\chi}$ is relevant for getting resonantly enhanced oscillations, which occur when

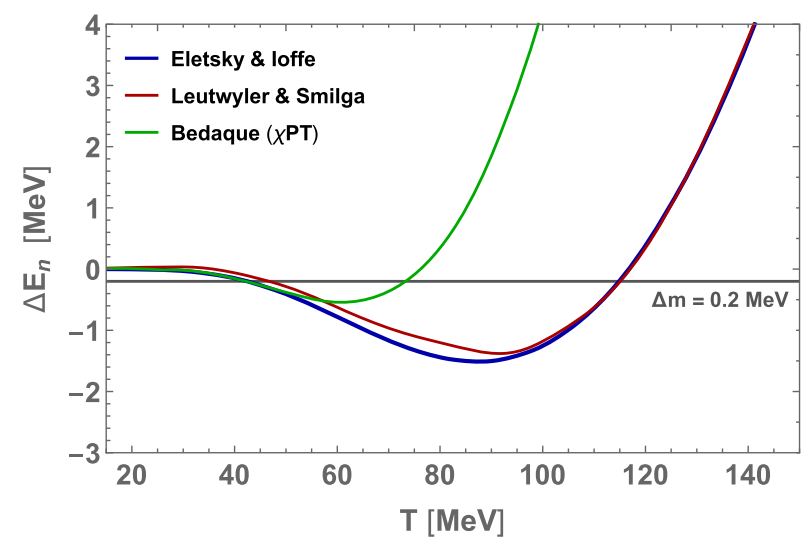

$\delta E \equiv \Delta m+\Delta E_{n}-\Delta E_{\chi}$ vanishes and the eigenvalues of $\mathcal{H}$ become degenerate. For illustration, we plot in Fig. 1 (right panel) the difference in thermal self-energies, with $g^{\prime} \xi=0,0.5,1$, along with a fiducial value of $\Delta m=$ $0.2 \mathrm{MeV}$. Clearly there are two resonant temperatures, where the curves cross the horizontal line. We will show that baryon production is dominated by the resonance at the lower temperature.

\section{A. Heuristic approach}

To find the efficiency of $\chi \rightarrow n$ oscillations for producing the baryon asymmetry, we first use an approximate formalism [42] that has the advantage of being simple and intuitive. Diagonalizing $\mathcal{H}$ leads to the mixing angle $\theta$,

$$
\tan (2 \theta)=\frac{2 \delta m}{\Delta m+\Delta E_{n}-\Delta E_{\chi}} \equiv \frac{2 \delta m}{\delta E} .
$$

The difference between the eigenvalues is given by

$$
|\delta \omega|=\sqrt{(\delta E)^{2}+4 \delta m^{2}} .
$$

Imagine starting with a state that is purely $|\chi\rangle$ at $t=0$. Evolving it with the Hamiltonian $\mathcal{H}$ in Eq. (6) leads to

$$
\begin{aligned}
|\psi(t)\rangle= & e^{-i\left(\omega_{1}+\omega_{2}\right) t / 2}\left[\left(\cos \frac{\delta \omega}{2} t-i \cos 2 \theta \sin \frac{\delta \omega}{2} t\right)|\chi\rangle\right. \\
& \left.-i\left(\sin 2 \theta \sin \frac{\delta \omega}{2} t\right)|n\rangle\right] .
\end{aligned}
$$

Therefore, the probability to oscillate into a neutron is given by

$$
P_{n}(t)=\sin ^{2}(2 \theta) \sin ^{2}(\delta \omega t / 2) .
$$

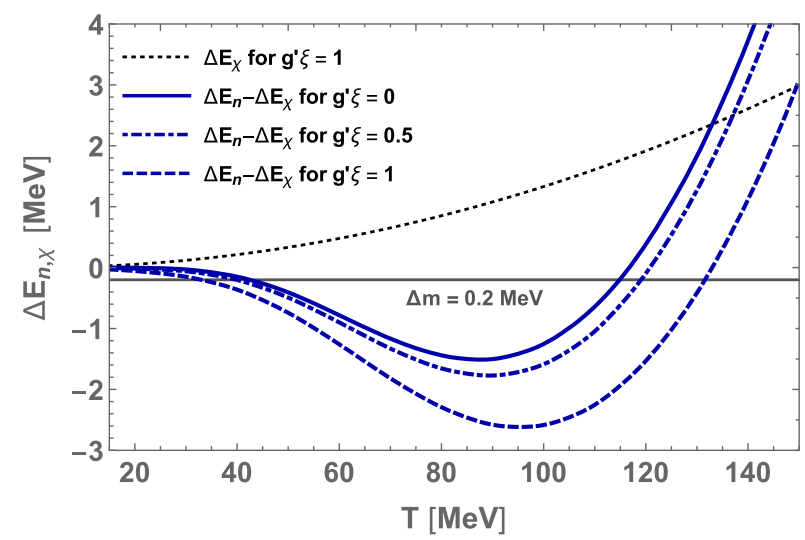

FIG. 1. Left panel: Thermal shifts $\Delta E_{n}$ to neutron mass from dispersion relations [36,37] (purple, blue) and chiral perturbation theory [38] (green). Right panel: Effect of including the DM thermal mass. The dotted curve is $\Delta E_{\chi}$ for $g^{\prime} \xi=1$ from Eq. (7). Lower curves show the difference in the thermal self-energies, $\Delta E_{n}-\Delta E_{\chi}$, for $g^{\prime} \xi=0,0.5$, 1. For comparison, we also indicate the maximal mass difference $\Delta m \equiv m_{n}-m_{\chi} \lesssim 0.2 \mathrm{MeV}$ compatible with baryon asymmetry generation and the stability of ${ }^{9} \mathrm{Be}$ and ${ }^{11} \mathrm{Be}$ through $n \rightarrow \chi+\gamma$; resonantly enhanced oscillations occur for $\Delta E_{n}-\Delta E_{\chi} \cong-\Delta m_{\chi}$. 
Because of the large rate of interactions $\Gamma_{n}$ of neutrons on heat bath pions, however, there may not be time for a full oscillation. In general, one should carry out a time average over the short timescale $1 / \Gamma_{n}$ :

$$
\bar{P}_{n}=\Gamma_{n} \int_{0}^{\infty} d t e^{-\Gamma_{n} t} P_{n}(t)=\frac{2 \delta m^{2}}{\delta \omega^{2}+\Gamma_{n}^{2}},
$$

where we use $\sin ^{2} 2 \theta=\delta m^{2} /\left(\delta m^{2}+\delta E^{2} / 4\right)$.

The rate of production of neutrons via oscillations is then $\Gamma_{\text {osc }}=\bar{P}_{n} \Gamma_{n}$ per $\chi$ particle (ignoring the much smaller elastic scattering rate $\Gamma_{\chi}$ of DM on dark photons and scalars). ${ }^{4}$ The inverse process of $\chi$ production must proceed with the same rate per neutron, so the number density of $\mathrm{DM}$ overall decreases as $\dot{n}_{\chi}=-\Gamma_{\text {osc }}\left(n_{\chi}-n_{n}\right)$. Since baryon number, and hence the total number of $\chi$ 's plus neutrons, is conserved, the equation determining the fraction $f=n_{n} /\left(n_{\chi}+n_{n}\right)$ of DM converting to neutrons is thus

$$
\dot{f}=\Gamma_{\mathrm{osc}}(1-2 f),
$$

which has the solution

$$
\begin{aligned}
f & =\frac{1}{2}\left(1-\exp \left(-2 \int d t \Gamma_{\text {osc }}\right)\right) \\
& \cong \frac{1}{2}\left(1-\exp \left(-2 \int \frac{d T}{T} \frac{\Gamma_{n} \bar{P}_{n}}{H}\right)\right)
\end{aligned}
$$

(with $H \cong 1.66 \sqrt{g_{*}} T^{2} / M_{p}, M_{p}=1.22 \times 10^{19} \mathrm{GeV}$, and $g_{*} \cong 10.75$ ), so long as $P_{\text {osc }}<1$. We need

$$
f \cong 0.16
$$

to get the desired abundance ratio $\Omega_{\chi} / \Omega_{B}=(1-f) / f \cong$ 5.3 , dictated by the coincidence that $m_{\chi} \cong m_{n}$.

It remains for us to determine the rate $\Gamma_{n}$. The cross section for neutrons to scatter on pions at low energy is $[43,44]^{5}$

$$
\sigma_{n \pi}=4 \pi a_{0}^{2} \cong \frac{0.1}{m_{\pi}^{2}} \cong 2 \mathrm{mb},
$$

giving the rate

$$
\Gamma_{n}=n_{\pi}\left\langle\sigma_{n \pi} v\right\rangle,
$$

with $\langle v\rangle=\sqrt{8 T /\left(\pi m_{\pi}\right)} \cong 1.6 \sqrt{T / m_{\pi}}$, where $n_{\pi}$ is the thermal density of pions, including a factor of 3 for isospin multiplicity.

\footnotetext{
${ }^{4}$ The cross section for $\phi \chi$ scattering from the interaction (47) is $10^{-7} \mathrm{mb}$, saturating the bound (50). For $m_{\phi}=60 \mathrm{MeV}$, for example, $n_{\phi} / n_{\pi}$ is only $\sim 7$ at the resonance temperature. See also Appendix A.

${ }^{5}$ We average the contributions from the $I=1 / 2$ and $3 / 2$ isospin scattering lengths as $\sigma=4 \pi\left(a_{1 / 2}^{2}+2 a_{3 / 2}^{2}\right) / 3$.
}

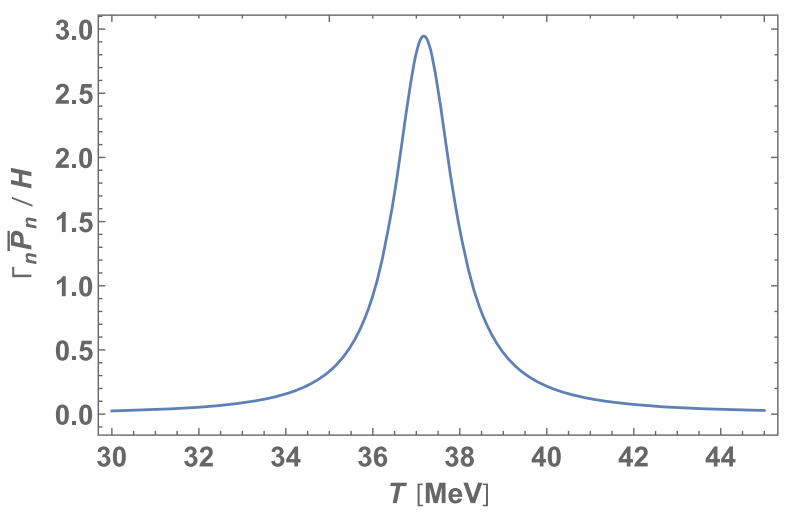

FIG. 2. The integrand $\Gamma_{n} \bar{P}_{n} / H$ of Eq. (14) versus the photon temperature $T$, showing how baryon number generation is dominated by the resonant enhancement of $\chi-n$ oscillations, using the fiducial parameters $\Delta m=0.105 \mathrm{MeV}$ and $\delta m=$ $1.05 \times 10^{-10} \mathrm{MeV}$ of Eq. (22).

This formalism makes it transparent that baryon production is dominated by the resonant enhancement of the oscillations by the finite- $T$ contributions to $\delta E$, which allow for $\delta E\left(T_{r}\right)=0$ at the resonance temperature $T_{r}$. At $T=0$, tight constraints on $\Delta m$ and $\delta m$ (to be discussed below) imply a vacuum mixing angle $\theta=2 \delta m / \Delta m$ that is by far too small to allow oscillations efficient enough for baryogenesis, but at $T_{r}$ the mixing is maximal, although the oscillation probability is reduced by the damping from $\Gamma_{n}$ in Eq. (12). This is illustrated in Fig. 2 for a benchmark model, Eq. (22) below, for which $T_{r} \cong 37 \mathrm{MeV}$. Because of the damping, the final efficiency for baryon production scales as $\delta m^{2} / \Gamma_{n}$.

\section{B. Boltzmann equations}

A more rigorous approach is to solve Boltzmann equations for the relative abundances, including off-diagonal terms arising from the density matrix, that take into account the oscillations. The formalism is described for oscillations of DM and its antiparticle in Refs. [45,46], and it requires only small modifications for neutron-DM oscillations. In terms of the independent variable $x \equiv m_{n} / T$, the equation for the matrix of abundances $Y=n / s$ is ${ }^{6}$

$$
\begin{aligned}
\frac{d Y}{d x}= & -\frac{i}{H x}[\mathcal{H}, Y]-\frac{\Gamma_{n}}{2 H x}\left[P_{n},\left[P_{n}, Y\right]\right]-\frac{\Gamma_{\chi}}{2 H x}\left[P_{\chi},\left[P_{\chi}, Y\right]\right] \\
= & -\frac{i}{H x}\left(\begin{array}{cc}
-\delta m Y_{-} & -\delta m \delta Y+\delta E Y_{12} \\
\delta m \delta Y-\delta E Y_{21} & \delta m Y_{-}
\end{array}\right) \\
& -\frac{\Gamma_{n}+\Gamma_{\chi}}{2 H x}\left(\begin{array}{cc}
0 & Y_{12} \\
Y_{21} & 0
\end{array}\right),
\end{aligned}
$$

\footnotetext{
${ }^{6}$ Here $s$ is taken to be the entropy in the visible sector. Since baryon number $Y_{1}+Y_{2}$ is conserved, we are only concerned with the fraction $f$ of DM that converts to baryons, from which $s$ divides out.
} 
where we define $\delta Y \equiv Y_{11}-Y_{22}=Y_{n}-Y_{\chi}, \quad Y_{ \pm}=$ $Y_{12} \pm Y_{21}$, and $P_{n, \chi}$ projects onto the states 1 (the neutron) or 2 (the $\chi$ ), respectively. As before, we will ignore $\Gamma_{\chi}$, since it is much smaller than $\Gamma_{n}{ }^{7}$

Since $\chi$ can be assigned a baryon number, which is conserved, the combination $Y_{11}+Y_{22}=Y_{n}+Y_{\chi}$ does not evolve. It is then convenient to recast the equations in terms of $\delta Y, Y_{+}$and $\eta_{-} \equiv i \delta m Y_{-}$:

$$
\begin{aligned}
\delta Y^{\prime} & =\frac{2}{H x} \eta_{-}, \\
\eta_{-}{ }^{\prime} & =\frac{\delta m}{H x}\left(-2 \delta m \delta Y+\delta E Y_{+}\right)-\frac{\Gamma_{n}}{2 H x} \eta_{-}, \\
Y_{+}{ }^{\prime} & =-\frac{\delta E}{\delta m H x} \eta_{-}-\frac{\Gamma_{n}}{2 H x} Y_{+} .
\end{aligned}
$$

This form has the advantage that all the functions are now real valued, and the coefficients are of order unity, for cases of interest. The rescaling of $Y_{-}$by $\delta m$ alleviates the problem of stiffness in the numerical integration. We integrate the system (19) with initial conditions $Y_{22}=$ $Y_{\chi}^{\infty}$ and $Y_{i j}=0$ for the other components. Since $Y_{11}+Y_{22}$ is conserved, the fraction of DM that converts to neutrons is $f=Y_{11} / Y_{\chi}^{\infty}=1-Y_{22} / Y_{\chi}^{\infty}$. We note that this fraction is necessarily independent of the initial DM abundance $Y_{\chi}^{\infty}$, so for any value of $f$ we can simply rescale $Y_{\chi}^{\infty}$ such that the observed DM density $\rho_{\mathrm{CDM}}=\operatorname{sm}_{\chi} Y_{11}^{0}$ is recovered at late times (in practice, we use $Y_{\chi}^{\infty}=1$ as the initial condition). Given that the DM and neutron are nearly degenerate, we can identify $f /(1-f)=\Omega_{b} / \Omega_{\mathrm{CDM}}$ at late times.

\section{Results}

Figure 3 shows the correlation between $\delta m$ and $\Delta m$ needed to get the observed baryon asymmetry, by fixing $f$ as stated in Eq. (15), for several values of $g^{\prime} \xi$. Each curve is doubled, with a small splitting, showing the good agreement between the heuristic treatment (lower) and the Boltzmann equation (upper). We noted above that the baryon asymmetry scales as $\delta m^{2} / \Gamma_{n}$. For larger $\Delta m$, the resonance temperature is increased, and the rate of $\Gamma_{n}$ is larger due to the higher density of pions, explaining why $\delta m$ must increase with $\Delta m$ to keep the baryon asymmetry constant.

On the same figure, we plot the upper limit on the vacuum mixing angle from null searches for the decay $n \rightarrow$ $\chi \gamma$ (blue curve), whose branching ratio must be $\lesssim 0.17$ times the $0.9 \%$ needed to resolve the lifetime discrepancy [27]. This gives

\footnotetext{
${ }^{7}$ It is worth pointing out that the elastic scatterings on $n$ and $\chi$ enter equally into the damping of the oscillations, regardless of whether one considers $n \rightarrow \chi$ or $\chi \rightarrow n$ transitions. This is because either interaction can measure the state of the system, regardless of the outcome of the measurement.
}

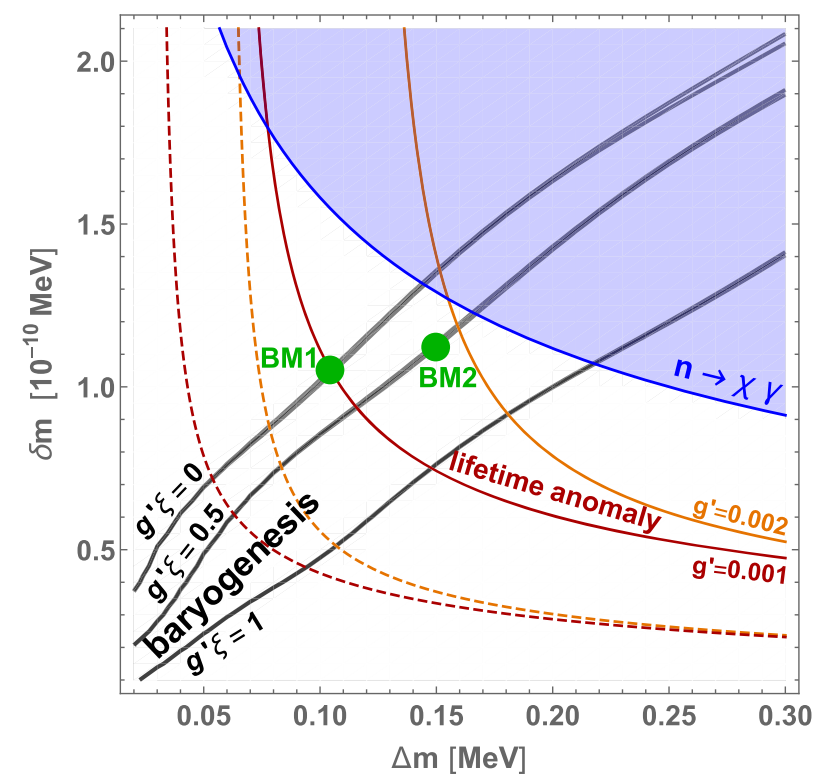

FIG. 3. Black contours give the right baryon asymmetry as in Eq. (15), in the $\delta m-\Delta m$ plane, for several values of $g^{\prime} \xi$. The differences between the baryogenesis predictions from the matrix Boltzmann equations (19) and the heuristic method given by Eq. (14) are not visible on this scale. The blue area is excluded from $n \rightarrow \chi \gamma$ decays (stability of ${ }^{9} \mathrm{Be}$ ). Red (orange) solid lines indicate the values of $\delta m$ needed to explain the neutron lifetime anomaly, assuming $m_{\gamma^{\prime}} / g^{\prime}=60 \mathrm{MeV}$ and $g^{\prime}=0.002$ (0.001). The lower dashed curves are the same, but assuming $m_{\gamma^{\prime}} / g^{\prime}=30 \mathrm{MeV}$.

$$
\delta m \lesssim 5 \times 10^{-11} \operatorname{MeV}\left(\frac{1 \mathrm{MeV}}{\Delta m}\right)^{1 / 2}
$$

Figure 3 thus implies that for reasonable values of $g^{\prime} \xi$, the baryon asymmetry can be explained only if $\Delta m \lesssim 0.2 \mathrm{MeV}$.

We next consider the neutron lifetime anomaly, for which the mixing mass should satisfy [25]

$$
\delta m=\frac{4.2 \times 10^{-13}}{\left(1-\left(m_{\gamma^{\prime}} / \Delta m\right)^{2}\right)^{3 / 4}}\left(\frac{m_{\gamma^{\prime}}}{g^{\prime}}\right)\left(\frac{1 \mathrm{MeV}}{\Delta m}\right)^{1 / 2} .
$$

Combining this with the limit on $m_{\gamma^{\prime}} / g^{\prime}$ from neutron stars in Eq. (2), this becomes a constraint on $\delta m$ as a function of $\Delta m$ and $g^{\prime}$ (with a slight dependence upon the assumed nuclear equation of state). Using $m_{\gamma^{\prime}} / g^{\prime}=60 \mathrm{MeV}$, we indicate in Fig. 3 the values of $\delta m$ that are required to resolve the lifetime anomaly for two representative values of $g^{\prime}=0.001,0.002$ (solid red and orange curves, respectively). It is possible to choose a smaller value of $m_{\gamma^{\prime}} / g^{\prime}$ [which is consistent with the neutron star constraint (2)], leading to the lifetime anomaly being resolved by smaller values of $\delta m$. The choice $m_{\gamma^{\prime}} / g^{\prime}=30 \mathrm{MeV}$ is plotted for illustration (dashed red and orange curves), for the same two values of $g^{\prime}$. This value of $m_{\gamma^{\prime}} / g^{\prime}=30 \mathrm{MeV}$ is still 
consistent with constraints on the DM self-interaction rate; see Sec. IV E below.

To find a consistent intersection of the baryon asymmetry and lifetime anomaly curves below the $n \rightarrow \chi \gamma$ constraint in Fig. 3, it is necessary to take $g^{\prime} \lesssim 0.002$. The neutron star constraint in Eq. (2) hence implies that the dark photon must be very light, $m_{\gamma^{\prime}} \lesssim 100 \mathrm{keV}$. Since $\xi<1$, the thermal self-energy of $\chi$ as given in Eq. (7) is furthermore irrelevant; this effectively places us on the baryon asymmetry curve corresponding to $g^{\prime} \xi=0$. As an example, the benchmark values

$$
\text { B1: } \begin{aligned}
\Delta m & =0.105 \mathrm{MeV}, \quad \delta m=1.05 \times 10^{-10} \mathrm{MeV}, \\
g^{\prime} & =0.001, \quad m_{\gamma^{\prime}}=60 \mathrm{keV}
\end{aligned}
$$

are compatible with all the constraints ( $\xi$ being in principle unconstrained but, as we will see shortly, uniquely determined by the decoupling temperature).

The dark photon mass in these scenarios is significantly below the $2 m_{e}$ threshold. In the absence of additional channels, the dominant decay mode would then be $\gamma^{\prime} \rightarrow 3 \gamma$, which is so slow that the initially thermally distributed dark photons would be metastable and overclose the Universe [35] (see Ref. [33] for additional constraints). This motivates us to introduce the additional massless Dirac neutrino $\nu^{\prime}$ already mentioned in Sec. II, enabling the much more efficient decay $\gamma^{\prime} \rightarrow \nu^{\prime} \bar{\nu}^{\prime}$.

Alternatively, if we ignore the neutron decay anomaly (assuming, for example, that it is due to experimental error) and insist only on low-scale baryogenesis, it is not necessary for $g^{\prime}$ to be small, nor for the dark photon mass to respect the kinematical constraint [Eq. (3)]. This in turn allows heavier dark photons and the possibility for fast $\gamma^{\prime} \rightarrow e^{+} e^{-}$decays through kinetic mixing, via the Lagrangian term $-(\epsilon / 2) F_{\mu \nu} F^{\prime \mu \nu}$, without the need to introduce additional light degrees of freedom like $\nu^{\prime} .8$

In this simpler scenario, the additional heavy fermion $\psi$ described in Sec. II is also no longer required, since with sizeable values of $g^{\prime}$ the process $\chi \bar{\chi} \rightarrow \gamma^{\prime} \gamma^{\prime}$ can efficiently annihilate away the symmetric DM component. Possible benchmark values for such a minimal baryogenesis scenario are

$$
\text { B2: } \begin{aligned}
\Delta m & =0.15 \mathrm{MeV}, \quad \delta m=1.12 \times 10^{-10} \mathrm{MeV}, \\
g^{\prime} \xi & =0.5, \quad m_{\gamma^{\prime}}=50 \mathrm{MeV} .
\end{aligned}
$$

\footnotetext{
${ }^{8}$ Some extra field carrying $\mathrm{U}(1)^{\prime}$ charge will be needed to insure $\mathrm{U}(1)^{\prime}$ charge neutrality of the Universe at early times, as we will discuss in Sec. IV A. However, it need not be light, and so will not necessarily contribute extra radiation degrees of freedom that contribute to $N_{\text {eff }}$. See Appendix D for more details.
}

In the $\mathbf{B} 2$ model, an upper bound on the kinetic mixing parameter $\epsilon$ comes from the scattering interactions of $\chi$ off protons, with a cross section

$$
\sigma_{\chi p}=\frac{4 \alpha\left(g^{\prime} \epsilon\right)^{2} \mu_{p \chi}^{2}}{m_{\gamma^{\prime}}^{4}},
$$

where $\mu_{p \chi}$ is the reduced mass of $\chi$ and the proton. Results from CRESST-III give the limit $g^{\prime} \epsilon \lesssim 1.2 \times 10^{-7}$ [47]. Even more constraining are the beam dump experiments at Orsay [48] and SLAC (E137) [49], which limit $\epsilon \gtrsim$ $1.5 \times 10^{-5}$ or $\epsilon \lesssim 4.2 \times 10^{-8}$ [50], and observations of the flux of neutrinos from supernova 1987A, which constrain $\epsilon \gtrsim 4.7 \times 10^{-8}$ or $\epsilon \leq 3.2 \times 10^{-10}$ [51].

The decay of the dark photon to electrons and positrons proceeds with a width and lifetime given by

$$
\begin{aligned}
\Gamma & =\frac{\alpha \epsilon^{2}}{3 m_{\gamma^{\prime}}}\left(m_{\gamma^{\prime}}^{2}+2 m_{e}^{2}\right)\left(1-4 m_{e}^{2} / m_{\gamma^{\prime}}^{2}\right)^{1 / 2}, \\
\tau & \cong 0.5\left(\frac{10^{-10}}{\epsilon}\right)^{2} \mathrm{~s} .
\end{aligned}
$$

The above constraints combine to give the limit $\tau \gtrsim 0.05 \mathrm{~s}$, which is sufficiently early to avoid disturbing the production of light elements during BBN [52,53]. As will be discussed in Sec. IV C, interactions other than the kinetic mixing can thermalize the dark and visible sectors, so there is no constraint on $\epsilon$ from this requirement.

\section{THERMAL HISTORY}

To have a fully consistent scenario, we need to address several issues: (1) redistribution of the presumed initial $\chi$ asymmetry into baryons should not have taken place at a higher temperature through the interactions present in our model, since this might be more important than the $\chi$ - $n$ oscillations; (2) the $\mathrm{U}(1)^{\prime}$-breaking transition where $\phi$ gets its VEV must occur before the oscillations go through resonance; (3) the additional light degrees of freedom in the dark sector must be compatible with constraints from BBN and $\mathrm{CMB}$; (4) the symmetric component of the $\chi$ relic density must be sufficiently suppressed, to avoid having more DM than is observed; and (5) the self-interaction cross section of $\chi$ should respect constraints from structure formation. We consider these in turn. The constraints (2)(5) are automatically satisfied for the benchmark point $\mathbf{B 2}$ if they are fulfilled for B1, so we adopt the $\mathbf{B} \mathbf{1}$ parameters in this section.

\section{A. Sequestering of $B$ at high $\boldsymbol{T}$}

Our assumption is that initial asymmetries in $\chi, \phi$, and $\nu^{\prime}$ were somehow created at a high temperature, while the standard model baryon and lepton asymmetries were initially vanishing. Asymmetries in $\phi$ or $\nu^{\prime}$ are needed in 
addition to that in $\chi$ to maintain $\mathrm{U}(1)^{\prime}$ charge neutrality of the Universe, since $\mathrm{U}(1)^{\prime}$ remains unbroken until $T \sim$ $100 \mathrm{MeV}$ (see Sec. IV B). However, the UV model of reference [33], augmented for the present purposes (see Sec. IV D), includes interactions which at high temperatures will redistribute these initial asymmetries into the other dark sector particles as well as into the visible sector. The relevant interactions are

$$
\begin{aligned}
\mathcal{L}_{\mathrm{UV}}= & \lambda_{1} \bar{d}^{a} P_{L} \chi \Phi_{1, a}+\lambda_{2} \epsilon^{a b c} \bar{u}_{a}^{C} P_{R} d_{b} \Phi_{2, c} \\
& +\lambda_{3} \bar{\chi} \phi \psi+\mu \phi \Phi_{1, a} \Phi_{2}^{* a}+\text { H.c. }
\end{aligned}
$$

$\lambda_{1,2,3}$ are dimensionless coupling constants, while $\mu$ is a constant with units of mass. $\Phi_{1}$ and $\Phi_{2}$ are both TeV-scale scalar fields that are colored triplets with baryon number $-2 / 3$, and $\Phi_{1}$ also caries $\mathrm{U}(1)^{\prime}$ charge. The resulting decays and inverse decays will be in equilibrium at high temperatures, redistributing any initial asymmetry amongst all particles in the plasma. Generically, this will induce an early baryon asymmetry of the same order as that in DM, contrary to our hypothesis that only neutron-DM oscillations are important.

To see this, it is sufficient to consider temperatures just below the electroweak phase transition (EWPT), $T_{c} \cong 160 \mathrm{GeV}$, at which the relevant $\mathrm{SM}$ interactions are sphalerons (still in equilibrium, since the EWPT is a crossover transition) and $W^{-} \leftrightarrow d \bar{u}, W^{-} \leftrightarrow e \bar{\nu}$. Following Ref. [54], we take the up- and down-type quarks of all generations to have common chemical potentials $\mu_{u}$ and $\mu_{d}$, and we denote the sum of neutrino chemical potentials by $\mu_{\nu}$. (The charged lepton potentials $\mu_{L}$ are eliminated in favor of $\mu_{W}$ and $\mu$.) At this scale, the effective operator from integrating out $\Phi_{i}$ is

$$
\frac{\lambda_{1} \lambda_{2} \mu}{m_{\Phi_{1}}^{2} m_{\Phi_{2}}^{2}} \phi \epsilon^{a b c}\left(\bar{u}_{a}^{C} P_{R} d_{b}\right)\left(\bar{\chi} P_{R} d_{c}\right),
$$

which is in equilibrium down to $T \cong 11 \mathrm{GeV}$ for a light dark scalar $\phi$ (see Appendix B). ${ }^{9}$ The coefficient of Eq. (27) is fixed, since it gives rise to the $n-\chi$ mass mixing term when $\phi$ gets its VEV,

$$
\delta m=\frac{\lambda_{1} \lambda_{2} \beta\langle\phi\rangle \mu}{m_{\Phi_{1}}^{2} m_{\Phi_{2}}^{2}} \sim 10^{-10} \mathrm{MeV}
$$

where $\beta=0.014 \mathrm{GeV}^{3}$ [55] from the lattice matrix element $\langle n|u d d| 0\rangle$.

The equilibrium constraints from Eq. (27) and the SM interactions [54] are then

\footnotetext{
${ }^{9}$ After this interaction no longer keeps $\chi$ in equilibrium, it will lead to $\chi$ decays before the QCD phase transition. However, the lifetime for such a decay is $\sim 10 \mathrm{~s}$ for our benchmark points, so the effect of these decays on the abundance of $\chi$ particles will be minimal.
}

$$
\begin{gathered}
\mu_{\chi}=\mu_{\phi}+\mu_{u}+2 \mu_{d}, \\
\mu_{W}=\mu_{d}-\mu_{u}, \\
0=9 \mu_{u}+6 \mu_{W}+\mu_{\nu} .
\end{gathered}
$$

These are supplemented by the vanishing of the conserved charges, electric and $\mathrm{U}(1)^{\prime}$,

$$
\begin{gathered}
Q \propto 3 \mu_{u}-\mu_{\nu}-9 \mu_{W}=0, \\
Q^{\prime} \propto \mu_{\chi}+\mu_{\phi}+Q_{\nu^{\prime}}^{\prime} \mu_{\nu^{\prime}}=0,
\end{gathered}
$$

where we have used that $Q_{\nu^{\prime}}^{\prime}$ is the $\nu^{\prime}$ charge relative to that of $\chi$ or $\phi$. These equations give quark asymmetries

$$
\mu_{u}=\frac{1}{11}\left(\mu_{\chi}-\mu_{\phi}\right), \quad \mu_{d}=5 \mu_{u}
$$

unless $\mu_{\chi}=\mu_{\phi}$ at this temperature. Such a cancellation does not come about without tuning the relative initial asymmetries of $\chi$ and $\nu^{\prime}$. How these chemical potentials are related to the initial asymmetries, possibly generated at a much higher scale, is discussed in Appendix C. The $\nu^{\prime}$ asymmetry is determined by Eq. (33) alone.

However, there is a simple low-energy mechanism for enforcing $\mu_{\phi}=\mu_{\chi}$, supposing there is a massive Majorana fermion $\psi^{\prime}$ in the dark sector with coupling

$$
\lambda^{\prime} \bar{\chi} \phi \psi^{\prime}+\text { H.c. }
$$

If in equilbrium, this gives the desired relation $\mu_{\phi}=\mu_{\chi}$, since $\mu_{\psi^{\prime}}=0$ by the Majorana nature of $\psi^{\prime}$. The decays $\psi^{\prime} \rightarrow \chi \phi$ are in equilbrium for $T \geq T_{c}=160 \mathrm{GeV}$, so long as

$$
\lambda^{\prime} \gtrsim 1 \times 10^{-7}\left(\frac{100 \mathrm{GeV}}{m_{\psi^{\prime}}}\right)^{1 / 2} .
$$

This supplies the extra condition needed to ensure that no baryon asymmetry is induced prior to the onset of $n-\chi$ oscillations.

The new interaction (35) is potentially dangerous, since it violates baryon number symmetry. The dominant process is the $\Delta B=2$ oscillation of $\chi-\bar{\chi}$ due to the mass term

$$
\frac{1}{2} \delta m_{\chi} \bar{\chi} \chi^{C}+\text { H.c. }=\frac{\lambda^{\prime 2} v^{\prime 2}}{2 m_{\psi^{\prime}}} \bar{\chi} \chi^{C}+\text { H.c. }
$$

arising after $\phi$ gets its $\operatorname{VEV} v^{\prime} / \sqrt{2}$ and integrating out $\psi^{\prime}$. In our scenario, this can be very small, $\delta m_{\chi} \cong 3 \times 10^{-18} \mathrm{MeV}$ for $v^{\prime}=60 \mathrm{MeV}$, as we have assumed, and $\lambda^{\prime}$ saturating the bound [Eq. (36)] with $m_{\psi^{\prime}}=100 \mathrm{GeV}$. Such oscillations have been studied in detail in Ref. [46]. They are damped by the scattering of $\chi$ on dark photons as long as the 
scattering rate $\Gamma_{s} \sim g^{\prime 4} T^{3} / m_{\gamma^{\prime}}^{2}$ exceeds the oscillation rate $2 \delta m_{\chi}$, true for temperatures

$$
T \gtrsim\left(\frac{2 \delta m_{\chi} m_{\gamma^{\prime}}^{2}}{g^{\prime 4}}\right)^{1 / 3} \sim 3 \mathrm{keV},
$$

which is far below the scales of interest for baryogenesis through $\chi-n$ oscillations. The estimate (38) applies for the B1 benchmark model, but it is even lower for $\mathbf{B 2}$.

\section{B. $\mathbf{U}(1)^{\prime}$-breaking phase transition}

The dark scalar $\phi$ must get its VEV before the $n-\chi$ oscillations begin, since the mass mixing term $\delta m$ coming from Eq. (27) is proportional to $\langle\phi\rangle$ in our model. Because the gauge coupling $g^{\prime}$ is very small, the phase transition in the dark sector is controlled by the dark scalar $\phi$ alone. Supposing its potential is

$$
V_{0}=\lambda_{\phi}\left(|\phi|^{2}-v^{2} / 2\right)^{2},
$$

the field-dependent masses for the real and imaginary parts of $\phi=\left(\varphi_{r}+i \varphi_{i}\right) / \sqrt{2}$ are

$$
\begin{aligned}
& m_{r}^{2}=\frac{\partial^{2} V}{\partial \varphi_{r}^{2}}=\lambda_{\phi}\left(6|\phi|^{2}-v^{\prime 2}\right), \\
& m_{i}^{2}=\frac{\partial^{2} V}{\partial \varphi_{i}^{2}}=\lambda_{\phi}\left(2|\phi|^{2}-v^{\prime 2}\right)
\end{aligned}
$$

(assuming that $\varphi_{r}$ is the component that gets the VEV). At mean-field level, the finite-temperature contribution to the potential is [56]

$$
V_{T}=\frac{T^{\prime 2}}{24}\left(m_{r}^{2}+m_{i}^{2}\right),
$$

where $T^{\prime}$ is the temperature of the dark sector. Combining Eqs. (39) and (41), one finds that the critical temperature, where the curvature of the full potential at $\phi=0$ vanishes, is given by

$$
T_{c}^{\prime}=\sqrt{3} v^{\prime}=\sqrt{3} \frac{m_{\gamma^{\prime}}}{g^{\prime}} \cong 104 \mathrm{MeV}
$$

independently of $\lambda_{\phi}$, so long as $\lambda_{\phi} \gg g^{\prime 2}$. Since $T^{\prime}=\xi T$ with $\xi<1$, we see that the critical temperature as measured in the visible sector is even larger, $T_{c}>104 \mathrm{MeV}$. This is comfortably above the resonance temperature $T_{3} \sim$ $30 \mathrm{MeV}$ for our benchmark model.

\section{Constraints on extra degrees of freedom}

The dark sector in our model has new light degrees of freedom at the scale of BBN, the $\sim 60 \mathrm{keV}$ dark photon and two massless Weyl neutrinos $\nu^{\prime}$, the latter being also present during temperatures relevant for the CMB. We show in Appendix B that the effective operator (27) keeps the two sectors in equilbrium until $T \cong 11 \mathrm{GeV}$, irrespective of the details of the UV model parameters. This relatively high decoupling temperature helps to reduce the number of extra radiation degrees of freedom, conventionally parametrized as the number of additional effective neutrino species, $\Delta N_{\text {eff }}$.

Following decoupling, the entropies in the two sectors are separately conserved. During the QCD transition, the relative temperature $\xi=T^{\prime} / T$ goes down, because the number of SM degrees of freedom, $g_{\mathrm{SM}}(11 \mathrm{GeV}) \simeq$ 86.25 , decreases by a greater factor than the corresponding decrease in the dark sector $\left(\chi+\nu^{\prime}+\phi+\gamma^{\prime} \rightarrow \nu^{\prime}+\gamma^{\prime}\right)$. We thus expect

$$
\xi_{\mathrm{BBN}} \cong\left(\frac{86.25}{10.75}\right)^{-1 / 3}\left(\frac{6.5}{11}\right)^{-1 / 3} \cong 0.59
$$

at $T>m_{e}$ when electrons are still in equilibrium. At this temperature, the dark photons and neutrinos are both present in the dark sector, and they contribute

$$
\Delta N_{\mathrm{eff}}=\left(\frac{4}{7} \times 3+2\right) \xi_{\mathrm{BBN}}^{4} \sim 0.47
$$

to the effective number of neutrino species. This is below the $3 \sigma$ bound $\Delta N_{\text {eff }}<0.54$, but larger than the $2 \sigma$ bound $\Delta N_{\text {eff }}<0.31$ allowed by BBN [57] (similar BBN limits are reported by Cyburt et al. [58]).

At lower temperatures, the number of relativistic degrees of freedom again changes in both sectors, as electrons and dark photons disappear. At the time of the CMB, we have a temperature ratio of

$$
\xi_{\mathrm{CMB}} \cong\left(\frac{10.75}{3.91} \times \frac{3.5}{6.5}\right)^{-1 / 3} \xi_{\mathrm{BBN}} \cong 0.52
$$

Taking into account that the temperature ratio of SM neutrinos and photons has also increased by that time, we hence get

$$
\Delta N_{\text {eff }}=2 \xi_{\mathrm{CMB}}^{4} \times(11 / 4)^{4 / 3} \sim 0.56 .
$$

This is above the $3 \sigma$ limit of $\Delta N_{\text {eff }}<0.45$ from Planck $[1,59]$, combining CMB and BAO measurements. But we note that this limit weakens to $\Delta N_{\text {eff }}<0.55(0.66)$ at $2 \sigma$ $(3 \sigma)$ [59] when adding the direct measurement of the Hubble rate, $H_{0}=(73.45 \pm 1.66) \mathrm{km} \mathrm{s}^{-1} \mathrm{Mpc}^{-1}$ [60], to these datasets.

For a more quantitative treatment, we implemented the full temperature dependence of the energy density for each dark sector d.o.f. in DarkSUSY [61], rather than the relativistic limit used in the above estimates, and plot the expected $\Delta N_{\text {eff }}$ as a function of decoupling temperature $T_{\text {dec }}$ in Fig. 4. The red line shows $\Delta N_{\text {eff }}$ at CMB times, while the blue line shows this quantity for $T=1 \mathrm{MeV}$; the 


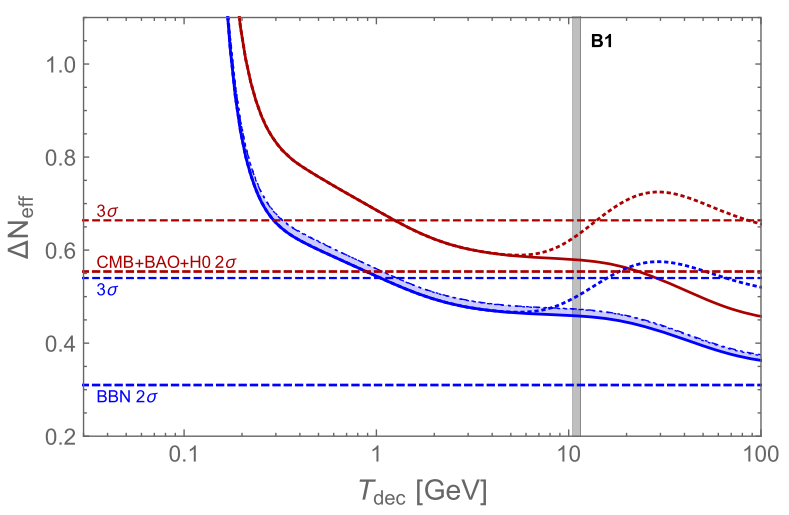

FIG. 4. The extra contributions to the effective number of neutrino species, $\Delta N_{\text {eff }}$, from the dark neutrinos $\nu^{\prime}$, the dark photon $\gamma^{\prime}$ (at $T \gtrsim 60 \mathrm{keV}$ ), and the dark Higgs boson $\phi$ (for $T \gtrsim 60 \mathrm{MeV}$ ). Dotted lines show the effect of adding a fermion with $m_{\psi}=50 \mathrm{GeV}$. The vertical line indicates the decoupling temperature $T_{\mathrm{dec}}$ for the benchmark point B1. We also indicate limits from BBN [57] and Planck data [1].

blue band corresponds to $\Delta N_{\text {eff }}$ for the whole range of temperatures $0.1 \mathrm{MeV}<T<5 \mathrm{MeV}$ most strongly probed by BBN. We also indicate in this plot the already mentioned constraints from BBN and the CMB. For the benchmark point B1, Fig. 4 confirms the above estimates for $\Delta N_{\text {eff }}$. At the same time, this figure illustrates the effect of changing $T_{\mathrm{dec}}$. For example, for a smaller $\langle\phi\rangle=m_{\gamma^{\prime}} / g^{\prime}$, the coefficient in front of Eq. (27) would increase, leading to a decrease in $T_{\text {dec }}$ that sharpens the tension with BBN and the CMB. A larger value of $\langle\phi\rangle$, on the other hand, cannot be achieved in view of the neutron star constraint [Eq. (2)]. To decrease the tension with $\Delta N_{\text {eff }}$ would thus require us to modify the low-energy operator given in Eq. (27) —or to make the dark photon and Higgs nonrelativistic already during $\mathrm{BBN}$, as in the benchmark point B2. Finally, if decoupling happens while some of the heavy degrees of freedom are still in equilibrium, this will increase the entropy ultimately dumped into $\nu^{\prime}$; we illustrate this in Fig. 4 by showing the effect of an additional fermion with mass $m_{\psi}=50 \mathrm{GeV}$ (dotted lines).

\section{Dark matter annihilation and kinetic decoupling}

Although we are assuming that the asymmetric component of the DM abundance is generated by an unknown mechanism, the symmetric (thermal) component is determined by the annihilations of $\chi \bar{\chi}$ into lighter particles. Because of the small value of $g^{\prime}=10^{-3}$, the cross sections for $\chi \bar{\chi} \rightarrow \gamma^{\prime} \gamma^{\prime}$ and $\chi \bar{\chi} \rightarrow \nu^{\prime} \bar{\nu}^{\prime}$ are inadequate for avoiding overclosure. However, the channel $\chi \bar{\chi} \rightarrow \phi \phi^{*}$ becomes available if we introduce a neutral Dirac fermion $\psi$ with coupling

$$
\lambda_{3} \bar{\chi} \phi \psi+\text { H.c. }
$$

This is consistent with a stability of $\chi$ as long as $m_{\psi}>m_{\chi}$. The cross section for $\chi \bar{\chi} \rightarrow \phi \phi^{*}$ by $\psi$ exchange, to leading order in the center-of-mass velocity $v_{\mathrm{cm}}$ of the DM and for $m_{\psi} \gg m_{\chi}$, is

$$
\sigma v_{\text {rel }}=\frac{\lambda_{3}^{4} v_{\mathrm{cm}}^{2}}{16 \pi m_{\psi}^{2}}
$$

After thermally averaging, we find that to avoid overclosure, one needs $m_{\psi} \lambda_{3}^{-2} \lesssim 1 \mathrm{TeV}$ [62].

For a more quantitative estimate, one needs to take into account the nonstandard temperature evolution in the dark sector. To this end, we implemented the exact $\xi(T)$ dependence as well as the full expressions for $\sigma v_{\text {rel }}$ in DarkSUSY. Solving the Boltzmann equation numerically for input parameters in the range of interest, we find that the following provides a reliable fit to the relic density of the symmetric component in the absence of an asymmetric component:

$$
\left(\Omega h^{2}\right)_{\chi+\bar{\chi}} \simeq 0.19\left(\frac{m_{\psi} / \lambda_{3}^{2}}{500 \mathrm{GeV}}\right)^{1.82} .
$$

The additional presence of the asymmetric component, however, makes the annihilation of $\bar{\chi}$ more efficient. From Fig. 4 in Ref. [62], we can read off that already an annihilation rate $\langle\sigma v\rangle$ a factor of 2 larger than the cross section $\langle\sigma v\rangle_{0}$ that would give the correct relic density in the purely symmetric case-i.e., in accordance with Eq. (49) results in a suppression of the symmetric component by a factor of almost 50 . Choosing this reference relic density as $\left(\Omega h^{2}\right)_{\chi+\bar{\chi}}=0.145$ [rather than the standard value of $\left(\Omega h^{2}\right)_{\mathrm{DM}} \simeq 0.12$, because $17 \%$ of the DM will convert to neutrons], this translates to the requirement

$$
\frac{m_{\psi}}{\lambda_{3}^{2}} \lesssim 300 \mathrm{GeV}
$$

This condition is easily satisfied as long as $\lambda_{3}$ is not too small (note that decreasing $m_{\psi}$ below about $100 \mathrm{GeV}$ would increase the tension with $\Delta N_{\text {eff }}$, cf. Fig. 4).

Even after freeze-out, both the symmetric and the asymmetric DM components are kept in local (kinetic) equilibrium with $\nu^{\prime}$. This is exactly one of the scenarios studied in Ref. [63] where, due to the presence of a light mediator, elastic scattering between $\chi$ and $\nu^{\prime}$ is highly efficient, and kinetic decoupling therefore happens significantly later than for standard WIMP candidates. The dark acoustic oscillations in the $\chi-\nu^{\prime}$ fluid during and after decoupling lead to a cutoff in the power spectrum of matter density perturbations with an associated mass scale of

$$
M_{\mathrm{cut}} \simeq 2 \times 10^{9} M_{\odot}\left(\frac{\xi}{0.5}\right)^{\frac{9}{2}} Q_{\nu^{\prime}}^{\frac{3}{2}}\left(\frac{g^{\prime}}{0.001}\right)^{3}\left(\frac{m_{\gamma^{\prime}}}{60 \mathrm{keV}}\right)^{-3} .
$$


A warm DM candidate with mass $\left[10^{11} h^{-1} M_{\odot} /\right.$ $\left.M_{\text {cut }}\right]^{1 / 4} \mathrm{keV}$ generates an almost identical small-scale suppression of the nonlinear power spectrum [64]. This implies that Lyman- $\alpha$ constraints on our model can be easily evaded as long as the charge of the dark sector neutrino is not too large, $Q_{\nu^{\prime}} \lesssim 1$ [65-67]. For $Q_{\nu^{\prime}} \sim 1$, in fact, the resulting mild suppression of the power spectrum might help to alleviate the missing satellites problem $[63,68]$.

\section{E. Dark matter self-interactions}

The cross section for $\chi \chi \rightarrow \chi \chi$ scattering is nonperturbatively enhanced by multiple $\gamma^{\prime}$ exchange, and hence cannot be calculated in the Born limit. We use DarkSUSY to calculate the transfer cross section for a repulsive Yukawa potential in the classical limit $m_{\chi} v_{\text {rel }} \gg m_{\gamma^{\prime}}$, based on parametrizations from Ref. [69], averaged over a Maxwellian velocity distribution with a most probable velocity $v_{0}$. For our benchmark model (22) and $v_{0}=30 \mathrm{~km} / \mathrm{s}$, this leads to $\left\langle\sigma_{T}\right\rangle / m_{\chi} \sim$ $0.15 \mathrm{~cm}^{2} / \mathrm{g}$, which may be strong enough to visibly affect the structure of dwarf galaxies [70-72]. The cross sections required to fully address the small-scale structure problems of cold DM are typically quoted to be a bit larger. This can be achieved by choosing a slightly smaller dark photon mass: for $m_{\gamma^{\prime}}=30 \mathrm{keV}$, for example, still respecting the constraint on $M_{\text {cut }}$ implied by Eq. (51), we find $\left\langle\sigma_{T}\right\rangle / m_{\chi} \sim 0.6 \mathrm{~cm}^{2} / \mathrm{g}$. The dependence of the transfer cross section on $v_{0}$ is shown in Fig. 5. The self-interaction rate drops very sharply with the typical velocity, implying that constraints on cluster scales [73-75] are not relevant for our model.

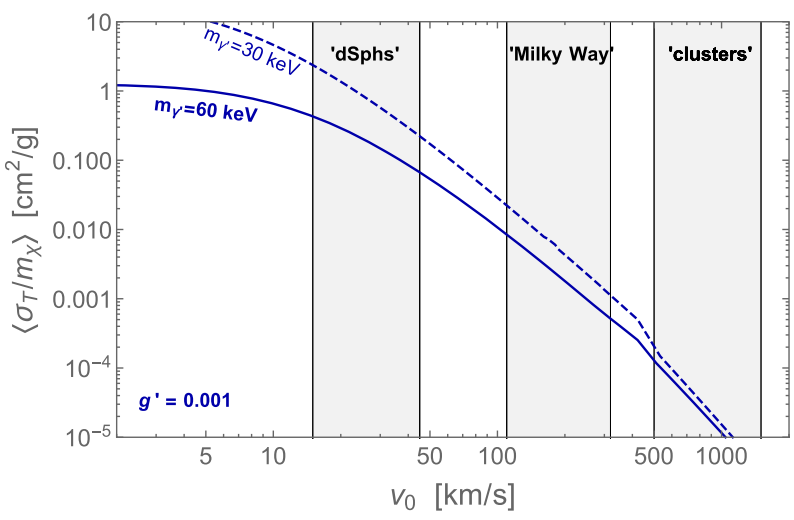

FIG. 5. Phase-space averaged self-interaction cross section over mass, $\left\langle\sigma_{T}\right\rangle / m_{\chi}$, versus characteristic DM velocity $v_{0}$, for $g^{\prime}=$ 0.001 and $m_{\gamma^{\prime}}=60 \mathrm{keV}$ (solid) or $m_{\gamma^{\prime}}=30 \mathrm{keV}$ (dashed). The vertical bands indicate typical velocities encountered in dwarf spheroidal galaxies, Milky Way-sized galaxies, and clusters. Mitigating the $\Lambda \mathrm{CDM}$ small-scale problems requires $\left\langle\sigma_{T}\right\rangle / m_{\chi} \sim$ $0.1-1 \mathrm{~cm}^{2} / \mathrm{g}$ at dwarf scales.

\section{CONCLUSIONS}

We have demonstrated that baryogenesis, starting from an initial dark matter asymmetry, can be successfully implemented through dark matter-neutron oscillations. It is intriguing that the same model invented to address the neutron lifetime anomaly in Ref. [33] is capable, with small modifications, of producing the observed baryon asymmetry in this way. A key requirement is that a somewhat smaller mass splitting, $\Delta m=m_{n}-m_{\chi} \sim 0.1 \mathrm{MeV}$, is now needed, relative to resolving only the lifetime anomaly. This arises because the baryogenesis curves in Fig. 3 fall in the region excluded by $n \rightarrow \chi \gamma$ limits at larger $\Delta m$. Simultaneously satisfying the neutron lifetime anomaly requires small $g^{\prime} \sim 10^{-3}$ and a consequently lower dark photon mass, $m_{\gamma}^{\prime} \sim 60 \mathrm{keV}$, mandating an additional dark radiation species $\nu^{\prime}$ so that dark photons can decay fast enough to avoid problems with $\mathrm{BBN}$ and the CMB. Intriguingly, the combination of dark radiation and a very light dark photon may help to alleviate known issues of $\Lambda \mathrm{CDM}$ cosmology at small scales.

The most ambitious version of our scenario, where both baryogenesis and the neutron lifetime anomaly are treated, is challenged by extra radiation contributing to $N_{\text {eff }}$, being near the edge of current cosmological constraints. Updated BBN constraints and future CMB observations, like the planned Simons observatory [76], are likely to provide the most sensitive experimental test of the proposal. On the other hand, no dark radiation is needed in a simpler version, exemplified by the parameter choices [Eq. (23)], that provides only baryogenesis but no significant dark decay channel for the neutron. In this version, new physics signals could arise from kinetic mixing of the photon with the dark photon. In both cases, it is possible that the heavy scalar triplets required by the UV-complete version of the model may be accessible at the LHC.

Let us stress in closing that the main phenomenon, of asymmetry sharing through $n-\chi$ oscillations, depends only upon the existence of $n-\chi$ mass mixing at low energies. It is remarkable that purely standard model physics, giving the neutron a negative self-energy correction at finite temperature, makes it possible for the oscillations to be resonant at temperatures not far above BBN. To make this part of a coherent picture including dark matter generation in the early Universe requires lifting the effective theory to its UV completion. It could be interesting to find other examples of microscopic models leading to these phenomena.

\section{ACKNOWLEDGMENTS}

We thank F. Kahlhoefer and K. Kainulainen for valuable discussions. This work was performed in part at the Aspen Center for Physics, which is supported by National Science Foundation Grant No. PHY-1607611. T. B. wishes to thank McGill University, where part of this manuscript was completed, for generous support and hospitality. J. M. C. 

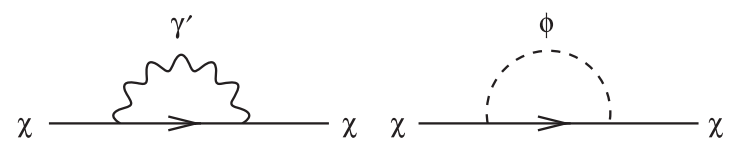

FIG. 6. Thermal self-energy corrections to $\chi$, where the intermediate boson has a finite-temperature propagator.

and J.M.C. gratefully acknowledge funding from the Natural Sciences and Engineering Research Council of Canada (NSERC).

\section{APPENDIX A: THERMAL SELF-ENERGY OF $\chi$}

The thermal contributions to the $\chi$ self-energy are shown in Fig. 6, in which the propagators of the intermediate light bosons are replaced by their thermal corrections (see, for example, Ref. [41]),

$$
\frac{1}{p^{2}-m^{2}} \rightarrow \frac{2 \pi \delta\left(p^{2}-m^{2}\right)}{e^{E / T}-1} .
$$

Evaluating the contribution from the hidden photon $\gamma^{\prime}$ gives

$$
\Sigma_{\chi}=g^{2} \int \frac{d^{4} p}{(2 \pi)^{3}} \gamma^{\mu} \frac{\delta\left(p^{2}-m_{\gamma^{\prime}}^{2}\right)}{\not \ell+\not p-m_{\chi}} \gamma^{\nu} \frac{-\eta_{\mu \nu}+p_{\mu} p_{\nu} / m_{\gamma^{\prime}}^{2}}{e^{\left|p_{0}\right| / T_{\gamma^{\prime}}}-1},
$$

which simplifies in the limit $\vec{k} \rightarrow 0$ of nonrelativistic $\chi$, and neglecting the dark photon mass, $m_{\gamma^{\prime}} \ll T_{\gamma^{\prime}}$. We get

$$
\Delta E_{\chi}=\Sigma_{\chi} \cong g^{\prime 2} \frac{T_{\gamma^{\prime}}^{2}}{8 m_{\chi}}
$$

in this limit.

A similar contribution to $\Delta E_{\chi} \sim \lambda_{3}^{2} / 8 m_{\Psi}$ arises when introducing the additional fermion $\psi$, with a coupling to $\chi$ given in Eq. (47). For large values of $g^{\prime}$, this contribution is parametrically suppressed by a factor of $m_{\chi} / m_{\psi}$ with respect to Eq. (A3). If $g^{\prime} \ll \lambda_{3}$, on the other hand, this can in fact be the dominant contribution to the thermal self-energy of $\chi$, but it would still be so small that it would not affect any of our numerical results (in Fig. 1, in particular, we would still remain on the curve labeled " $\xi g^{\prime}=0$ "). In our analysis, we therefore neglect the contribution from $\psi$ to $\Delta E_{\chi}$.

\section{APPENDIX B: DECOUPLING OF THE DARK SECTOR}

We estimate the freeze-out temperature for the dimension-7 interaction [Eq. (27)] from the scattering process $\phi \chi \rightarrow u d d$. At $T \gg m_{\chi}$, we can approximate all particles as being massless, with momenta $p_{1}=p_{\chi}, p_{2}=p_{\phi}$, and $p_{3,4,5}$ for the final-state quarks. The matrix element is

$$
\left\langle|\mathcal{M}|^{2}\right\rangle=\frac{6}{\Lambda^{6}} p_{1} \cdot p_{3} p_{4} \cdot p_{5}
$$

where $1 / \Lambda^{3} \cong 1.7 \times 10^{-10} \mathrm{GeV}^{-3}$ is the coefficient of the operator [Eq. (27)]. We can evaluate the cross section in the center-of-mass frame, choosing the 3-momenta to be

$$
\begin{gathered}
\vec{p}_{1}=E_{1}\left(s_{\alpha} c_{\phi}, s_{\alpha} s_{\phi}, c_{\alpha}\right), \\
\vec{p}_{2}=-\vec{p}_{1}, \\
\vec{p}_{3}=E_{3}(0,0,1), \\
\vec{p}_{4}=E_{4}\left(s_{\gamma}, 0, c_{\gamma}\right), \\
\vec{p}_{5}=-\left(\vec{p}_{3}+\vec{p}_{4}\right),
\end{gathered}
$$

where the angle between $p_{3}$ and $p_{4}$ is fixed by energymomentum conservation,

$$
c_{\gamma}=1+\frac{s-2 \sqrt{s}\left(E_{3}+E_{4}\right)}{2 E_{3} E_{4}},
$$

and $E_{5}=\sqrt{s}-E_{3}-E_{4}$. Then $p_{1} \cdot p_{3} p_{4} \cdot p_{5}=s\left(1-c_{\alpha}\right) \times$ $E_{3}\left(\sqrt{s}-2 E_{3}\right) / 4$, and the cross section is given by

$$
\begin{aligned}
\sigma & =\frac{1}{16(2 \pi)^{4} s} \int d c_{\alpha} d \phi \int_{0}^{\sqrt{s} / 2} d E_{3} \int_{\sqrt{s} / 2-E_{3}}^{\sqrt{s} / 2} d E_{4}\left\langle|\mathcal{M}|^{2}\right\rangle \\
& =\frac{s^{2}}{1024 \pi^{3} \Lambda^{6}} .
\end{aligned}
$$

The thermal average is

$\langle\sigma v\rangle=\frac{1}{32 T^{5}} \int_{0}^{\infty} d s \sigma s^{3 / 2} K_{1}(\sqrt{s} / T)=\frac{9 T^{4}}{256 \pi^{3} \Lambda^{6}}$.

Multiplying by the thermal density of a complex scalar and equating the scattering rate to the Hubble rate yields the freeze-out temperature $T_{f}=11 \mathrm{GeV}$.

\section{APPENDIX C: RELATIONS BETWEEN CHEMICAL POTENTIALS AND INITIAL ASYMMETRIES}

In this appendix, we present a calculation of the chemical potentials of both standard model and dark sector species when there is no baryon-number-violating interaction with $\psi^{\prime}$ which sets $\mu_{\chi}=\mu_{\phi}$. After the electroweak phase transition, the chemical potentials of the particle species in equilibrium are given by the equations in Sec. IV A, with an additional relation derived from the fact that the difference between the total baryon number and the lepton number is conserved when $\psi^{\prime}$ is not present:

$$
2 \mu_{\chi}+B-L=2 \mu_{0} \text {. }
$$


Here $B=6\left(\mu_{u}+\mu_{d}\right)$ and $L=\mu_{\nu}+2 \mu_{\ell}$, where $\mu_{\ell}$ is the sum of the charged lepton chemical potentials. $\mu_{0}$ is the initial $\chi$ chemical potential.

From these equations, the chemical potentials of the various species can be determined in terms of the initial asymmetries:

$$
\begin{gathered}
B=\frac{18}{61}\left(2 \mu_{0}+Q_{\nu^{\prime}}^{\prime} \mu_{\nu^{\prime}}\right), \\
L=-\frac{25}{12} B, \quad 6 \mu_{W}=\frac{2}{3} B, \\
2 \mu_{\chi}=\frac{1}{122}\left(22 \mu_{0}-111 Q_{\nu^{\prime}}^{\prime} \mu_{\nu^{\prime}}\right), \\
2 \mu_{\phi}=\frac{1}{122}\left(-22 \mu_{0}-133 Q_{\nu^{\prime}}^{\prime} \mu_{\nu^{\prime}}\right) .
\end{gathered}
$$

The coefficients of the chemical potentials in the above expressions are chosen so that their ratios correspond to the ratios of the asymmetries, e.g., $2 \mu_{\chi} / B=\left(n_{\chi}-n_{\bar{\chi}}\right) /$ $\left(n_{B}-n_{\bar{B}}\right)$. If $Q_{\nu^{\prime}}^{\prime} \mu_{\nu^{\prime}}=-2 \mu_{0}$, then $B=L=\mu_{W}=0$, $2 \mu_{\chi}=2 \mu_{\phi}=2 \mu_{0}$, and the analysis of Sec. III directly applies. However, other values for the initial asymmetries can lead to a baryon asymmetry when these interactions freeze out that is greater than what is observed today. Such scenarios are not viable-the subsequent oscillations will only drive the ratio of baryon-to- $\chi$ asymmetries to 1 , rather than reducing the baryon asymmetry to the observed value.

\section{APPENDIX D: LIMIT ON THE $\nu^{\prime}$ MASS}

Generically, one needs to introduce dark sector particles $\nu^{\prime}$ (though not necessarily massless fermions) with a nonvanishing chemical potential, which are stable because they carry a charge $Q^{\prime} \neq 1$. This extra field is needed to ensure that the Universe is neutral in $\mathrm{U}(1)^{\prime}$ charge at early times. These new states $\nu^{\prime}$ should not be too heavy in order not to overclose the Universe. For the case of benchmark scenario B2, we can estimate this constraint by noting that the annihilation into $\gamma^{\prime} \gamma^{\prime}$ keeps both $\nu^{\prime}$ and $\chi$ in equilibrium, respectively, until they are nonrelativistic. This allows us to relate $\mu_{\nu}$ to $n_{\nu}-n_{\bar{\nu}}$ in the nonrelativistic limit until freeze-out at $T_{\nu \text {,fo }}$, after which the comoving number density stays constant. The same applies, correspondingly, to the $\chi$ particles. For chemical potentials of roughly the same size, $\left|\mu_{\chi} / \mu_{\nu}\right| \sim \mathcal{O}(1)$, we then find that $m_{\nu^{\prime}} \sim 100 \mathrm{MeV}$ is already sufficient to suppress $\rho_{\nu} / \rho_{\chi} \ll 1$ at late times. While the existence of the additional states $\nu^{\prime}$ is necessary for our baryogenesis scenario to work, they can thus be heavy enough to leave the phenomenology of $\mathbf{B 2}$ unaffected.
[1] N. Aghanim et al. (Planck Collaboration), Planck 2018 results: VI. Cosmological parameters, arXiv:1807.06209.

[2] K. M. Zurek, Asymmetric dark matter: Theories, signatures, and constraints, Phys. Rep. 537, 91 (2014).

[3] J. Shelton and K. M. Zurek, Darkogenesis: A baryon asymmetry from the dark matter sector, Phys. Rev. D 82, 123512 (2010).

[4] K. Aitken, D. McKeen, T. Neder, and A. E. Nelson, Baryogenesis from oscillations of charmed or beautiful baryons, Phys. Rev. D 96, 075009 (2017).

[5] Z. Berezhiani and L. Bento, Neutron-Mirror Neutron Oscillations: How Fast Might They Be?, Phys. Rev. Lett. 96, 081801 (2006).

[6] Z. Berezhiani and L. Bento, Fast neutron: Mirror neutron oscillation and ultrahigh energy cosmic rays, Phys. Lett. B 635, 253 (2006).

[7] Z. Berezhiani, More about neutron-mirror neutron oscillation, Eur. Phys. J. C 64, 421 (2009).

[8] Z. Berezhiani, Neutron lifetime puzzle and neutron-mirror neutron oscillation, arXiv:1807.07906.

[9] S. M. Barr, R. S. Chivukula, and E. Farhi, Electroweak fermion number violation and the production of stable particles in the early Universe, Phys. Lett. B 241, 387 (1990).
[10] S. M. Barr, Baryogenesis, sphalerons and the cogeneration of dark matter, Phys. Rev. D 44, 3062 (1991).

[11] D. B. Kaplan, A Single Explanation for Both the Baryon and Dark Matter Densities, Phys. Rev. Lett. 68, 741 (1992).

[12] S. B. Gudnason, C. Kouvaris, and F. Sannino, Dark Matter from new technicolor theories, Phys. Rev. D 74, 095008 (2006).

[13] M. R. Buckley and L. Randall, Xogenesis, J. High Energy Phys. 09 (2011) 009.

[14] M. Blennow, B. Dasgupta, E. Fernandez-Martinez, and N. Rius, Aidnogenesis via leptogenesis and dark sphalerons, J. High Energy Phys. 03 (2011) 014.

[15] B. Dutta and J. Kumar, Asymmetric dark matter from hidden sector baryogenesis, Phys. Lett. B 699, 364 (2011).

[16] P. Fileviez Pérez and H. H. Patel, Baryon asymmetry, dark matter and local baryon number, Phys. Lett. B 731, 232 (2014).

[17] R. Foot and R. R. Volkas, Was ordinary matter synthesized from mirror matter? An attempt to explain why $\Omega_{\mathrm{B}} \approx 0.2 \Omega_{\text {dark }}$, Phys. Rev. D 68, 021304 (2003).

[18] D. Hooper, J. March-Russell, and S. M. West, Asymmetric sneutrino dark matter and the $\Omega_{\mathrm{b}} / \Omega_{\mathrm{DM}}$ puzzle, Phys. Lett. B 605, 228 (2005).

[19] D. E. Kaplan, M. A. Luty, and K. M. Zurek, Asymmetric dark matter, Phys. Rev. D 79, 115016 (2009). 
[20] N. Haba and S. Matsumoto, Baryogenesis from dark sector, Prog. Theor. Phys. 125, 1311 (2011).

[21] P. Fileviez Perez and M. B. Wise, Baryon asymmetry and dark matter through the vector-like portal, J. High Energy Phys. 05 (2013) 094.

[22] G. Servant and S. Tulin, Baryogenesis and Dark Matter through a Higgs Asymmetry, Phys. Rev. Lett. 111, 151601 (2013).

[23] N. Bernal, C. S. Fong, and N. Fonseca, Sharing but not caring: Dark matter and the baryon asymmetry of the Universe, J. Cosmol. Astropart. Phys. 09 (2016) 005.

[24] G. Elor, M. Escudero, and A. Nelson, Baryogenesis and Dark Matter from $B$ Mesons, arXiv:1810.00880.

[25] B. Fornal and B. Grinstein, Dark Matter Interpretation of the Neutron Decay Anomaly, Phys. Rev. Lett. 120, 191801 (2018).

[26] F. E. Wietfeldt and G. L. Greene, Colloquium: The neutron lifetime, Rev. Mod. Phys. 83, 1173 (2011).

[27] Z. Tang et al., Search for the Neutron Decay $n \rightarrow X+\gamma$ where $X$ is a Dark Matter Particle, Phys. Rev. Lett. 121, 022505 (2018).

[28] X. Sun et al. (UCNA Collaboration), Search for dark matter decay of the free neutron from the UCNA experiment: $n \rightarrow \chi+e^{+} e^{-}$, Phys. Rev. C 97, 052501 (2018).

[29] D. McKeen, A. E. Nelson, S. Reddy, and D. Zhou, Neutron Stars Exclude Light Dark Baryons, Phys. Rev. Lett. 121, 061802 (2018).

[30] G. Baym, D. H. Beck, P. Geltenbort, and J. Shelton, Testing Dark Decays of Baryons in Neutron Stars, Phys. Rev. Lett. 121, 061801 (2018).

[31] T. F. Motta, P. A. M. Guichon, and A. W. Thomas, Implications of Neutron Star Properties for the Existence of Light Dark Matter, J. Phys. G 45, 05LT01 (2018).

[32] S. Gandolfi, J. Carlson, and S. Reddy, The maximum mass and radius of neutron stars and the nuclear symmetry energy, Phys. Rev. C 85, 032801 (2012).

[33] J. M. Cline and J. M. Cornell, Dark decay of the neutron, J. High Energy Phys. 07 (2018) 081.

[34] H. Ejiri and J. D. Vergados, Neutron disappearance inside the nucleus, J. Phys. G 46, 025104 (2019).

[35] J. Redondo and M. Postma, Massive hidden photons as lukewarm dark matter, J. Cosmol. Astropart. Phys. 02 (2009) 005.

[36] H. Leutwyler and A. V. Smilga, Nucleons at finite temperature, Nucl. Phys. B342, 302 (1990).

[37] V. L. Eletsky and B. L. Ioffe, On the thermal mass shift of nucleons, Phys. Lett. B 401, 327 (1997).

[38] P. F. Bedaque, Chiral perturbation theory analysis of baryon temperature mass shifts, Phys. Lett. B 387, 1 (1996).

[39] C. A. Dominguez and M. Loewe, Nucleon propagator at finite temperature, Z. Phys. C 58, 273 (1993).

[40] M. Kacir and I. Zahed, Nucleons at finite temperature, Phys. Rev. D 54, 5536 (1996).

[41] D. Notzold and G. Raffelt, Neutrino dispersion at finite temperature and density, Nucl. Phys. B307, 924 (1988).

[42] J. M. Cline, Constraints on Almost Dirac Neutrinos from Neutrino-Antineutrino Oscillations, Phys. Rev. Lett. 68, 3137 (1992).
[43] N. Fettes, U. G. Meissner, and S. Steininger, Pion-nucleon scattering in chiral perturbation theory: 1. Isospin symmetric case, Nucl. Phys. A640, 199 (1998).

[44] J. Ruiz de Elvira, M. Hoferichter, B. Kubis, and U. G. Meißner, Extracting the $\sigma$-term from low-energy pionnucleon scattering, J. Phys. G 45, 024001 (2018).

[45] M. Cirelli, P. Panci, G. Servant, and G. Zaharijas, Consequences of DM/antiDM oscillations for asymmetric WIMP dark matter, J. Cosmol. Astropart. Phys. 03 (2012) 015.

[46] S. Tulin, H. B. Yu, and K. M. Zurek, Oscillating asymmetric dark matter, J. Cosmol. Astrophys. Phys. 05 (2012) 013.

[47] F. Petricca et al. (CRESST Collaboration), First results on low-mass dark matter from the CRESST-III experiment, arXiv:1711.07692.

[48] M. Davier and H. Nguyen Ngoc, An unambiguous search for a light Higgs boson, Phys. Lett. B 229, 150 (1989).

[49] J. D. Bjorken, S. Ecklund, W. R. Nelson, A. Abashian, C. Church, B. Lu, L. W. Mo, T. A. Nunamaker, and P. Rassmann, Search for neutral metastable penetrating particles produced in the SLAC beam dump, Phys. Rev. D 38, 3375 (1988).

[50] S. Andreas, C. Niebuhr, and A. Ringwald, New limits on hidden photons from past electron beam dumps, Phys. Rev. D 86, 095019 (2012).

[51] J. H. Chang, R. Essig, and S. D. McDermott, Revisiting Supernova 1987A constraints on dark photons, J. High Energy Phys. 01 (2017) 107.

[52] M. Hufnagel, K. Schmidt-Hoberg, and S. Wild, BBN constraints on $\mathrm{MeV}$-scale dark sectors: Part II. Electromagnetic decays, J. Cosmol. Astropart. Phys. 11 (2018) 032.

[53] L. Forestell, D. E. Morrissey, and G. White, Limits from BBN on light electromagnetic decays, J. High Energy Phys. 01 (2019) 074.

[54] J. A. Harvey and M. S. Turner, Cosmological baryon and lepton number in the presence of electroweak fermion number violation, Phys. Rev. D 42, 3344 (1990).

[55] Y. Aoki, T. Izubuchi, E. Shintani, and A. Soni, Improved lattice computation of proton decay matrix elements, Phys. Rev. D 96, 014506 (2017).

[56] L. Dolan and R. Jackiw, Symmetry behavior at finite temperature, Phys. Rev. D 9, 3320 (1974).

[57] M. Hufnagel, K. Schmidt-Hoberg, and S. Wild, BBN constraints on MeV-scale dark sectors: Part I. Sterile decays, J. Cosmol. Astropart. Phys. 02 (2018) 044.

[58] R. H. Cyburt, B. D. Fields, K. A. Olive, and T. H. Yeh, Big bang nucleosynthesis: 2015, Rev. Mod. Phys. 88, 015004 (2016).

[59] (Planck Legacy Archive (PLA)), https://pla.esac.esa.int/ \#cosmology.

[60] A. G. Riess et al., New parallaxes of Galactic Cepheids from spatially scanning the Hubble Space Telescope: Implications for the Hubble constant, Astrophys. J. 855, 136 (2018).

[61] T. Bringmann, J. Edsjö, P. Gondolo, P. Ullio, and L. Bergström, DarkSUSY 6: An advanced tool to compute dark matter properties numerically, J. Cosmol. Astropart. Phys. 07 (2018) 033.

[62] M. L. Graesser, I. M. Shoemaker, and L. Vecchi, Asymmetric WIMP dark matter, J. High Energy Phys. 10 (2011) 110. 
[63] T. Bringmann, H. T. Ihle, J. Kersten, and P. Walia, Suppressing structure formation at dwarf galaxy scales and below: Late kinetic decoupling as a compelling alternative to warm dark matter, Phys. Rev. D 94, 103529 (2016).

[64] M. Vogelsberger, J. Zavala, F. Y. Cyr-Racine, C. Pfrommer, T. Bringmann, and K. Sigurdson, ETHOS: An effective theory of structure formation: Dark matter physics as a possible explanation of the small-scale CDM problems, Mon. Not. R. Astron. Soc. 460, 1399 (2016).

[65] V. Iršiš et al., New constraints on the free-streaming of warm dark matter from intermediate and small scale Lyman- $\alpha$ forest data, Phys. Rev. D 96, 023522 (2017).

[66] M. Garny, T. Konstandin, L. Sagunski, and S. Tulin, Lyman- $\alpha$ forest constraints on interacting dark sectors, J. Cosmol. Astropart. Phys. 09 (2018) 011.

[67] A. Garzilli, A. Magalich, T. Theuns, C. S. Frenk, C. Weniger, O. Ruchayskiy, and A. Boyarsky, The Lyman- $\alpha$ forest as a diagnostic of the nature of the dark matter, arXiv: 1809.06585 .

[68] R. Huo, M. Kaplinghat, Z. Pan, and H. B. Yu, Signatures of self-interacting dark matter in the matter power spectrum and the CMB, Phys. Lett. B 783, 76 (2018).

[69] F. Y. Cyr-Racine, K. Sigurdson, J. Zavala, T. Bringmann, M. Vogelsberger, and C. Pfrommer, ETHOS: An effective theory of structure formation: From dark particle physics to the matter distribution of the Universe, Phys. Rev. D 93, 123527 (2016).

[70] D. N. Spergel and P. J. Steinhardt, Observational Evidence for Self-Interacting Cold Dark Matter, Phys. Rev. Lett. 84, 3760 (2000).

[71] S. Tulin and H. B. Yu, Dark matter self-interactions and small scale structure, Phys. Rep. 730, 1 (2018).

[72] G. K. Karananas and A. Kassiteridis, Small-scale structure from neutron dark decay, J. Cosmol. Astropart. Phys. 09 (2018) 036.

[73] S. W. Randall, M. Markevitch, D. Clowe, A. H. Gonzalez, and M. Bradac, Constraints on the self-interaction crosssection of dark matter from numerical simulations of the merging galaxy cluster 1E 0657-56, Astrophys. J. 679, 1173 (2008).

[74] D. Harvey, R. Massey, T. Kitching, A. Taylor, and E. Tittley, The non-gravitational interactions of dark matter in colliding galaxy clusters, Science 347, 1462 (2015).

[75] M. Kaplinghat, S. Tulin, and H. B. Yu, Dark Matter Halos as Particle Colliders: Unified Solution to Small-Scale Structure Puzzles from Dwarfs to Clusters, Phys. Rev. Lett. 116, 041302 (2016).

[76] J. Aguirre et al. (Simons Observatory Collaboration), The Simons observatory: Science goals and forecasts, arXiv: 1808.07445 . 